\title{
Failure process of a bundle of plastic fibers
}

\author{
Frank Raischel, ${ }^{1, *}$ Ferenc Kun, ${ }^{2}$ and Hans J. Herrmann ${ }^{1,3}$ \\ ${ }^{1}$ ICP, University of Stuttgart, Pfaffenwaldring 27, D-70569 Stuttgart, Germany \\ ${ }^{2}$ Department of Theoretical Physics, University of Debrecen, P.O. Box 5, H-4010 Debrecen, Hungary \\ ${ }^{3}$ Departamento de Física, Universidade Federal do Ceará, Campus do Pici, 60451-970 Fortaleza CE, Brazil
}

(Received 13 January 2006; published 1 June 2006)

\begin{abstract}
We present an extension of fiber bundle models considering that failed fibers still carry a fraction $0 \leqslant \alpha$ $\leqslant 1$ of their failure load. The value of $\alpha$ interpolates between the perfectly brittle failure $(\alpha=0)$ and perfectly plastic behavior $(\alpha=1)$ of fibers. We show that the finite load bearing capacity of broken fibers has a substantial effect on the failure process of the bundle. In the case of global load sharing it is found that for $\alpha \rightarrow 1$ the macroscopic response of the bundle becomes perfectly plastic with a yield stress equal to the average fiber strength. On the microlevel, the size distribution of avalanches has a crossover from a power law of exponent $\approx 2.5$ to a faster exponential decay. For localized load sharing, computer simulations revealed a sharp transition at a well-defined value $\alpha_{c}$ from a phase where macroscopic failure occurs due to localization as a consequence of local stress enhancements, to another one where the disordered fiber strength dominates the damage process. Analyzing the microstructure of damage, the transition proved to be analogous to percolation. At the critical point $\alpha_{c}$, the spanning cluster of damage is found to be compact with a fractal boundary. The distribution of bursts of fiber breakings shows a power-law behavior with a universal exponent $\approx 1.5$ equal to the mean-field exponent of fiber bundles of critical strength distributions. The model can be relevant to understand the shear failure of glued interfaces where failed regions can still transmit load by remaining in contact.
\end{abstract}

DOI: 10.1103/PhysRevE.73.066101

PACS number(s): 46.50.+a, 62.20.Mk, 64.60.-i

\section{INTRODUCTION}

The failure of heterogeneous materials under various types of external loading conditions has attracted continuous scientific and technological interest during the past decade $[1,2]$. Both the macroscopic strength and the process of damaging of loaded specimens strongly depend on the disordered microscopic properties of the material. Hence, most of the theoretical studies are based on discrete models which can account for the disordered material properties and their interaction with the inhomogeneous stress field naturally arising in a damaged specimen. Fiber bundle models (FBM's) are one of the most important theoretical approaches in this field [3], which also served as the basis for the development of more complicated micromechanical models of fracture [4-6]. In spite of their simplicity, FBM's capture the most important ingredients of the failure process and make it also possible to obtain several characteristic quantities of high interest in closed analytic form. Based on FBM's important results have been obtained for the macroscopic response of the loaded specimen [7] and for the temporal [8-11] and spatial structure of damage on the microlevel. In the framework of FBM's the analogy of fracture and critical phenomena [12-15] has also been addressed, which is of high practical importance for the forecasting of imminent failure of loaded systems $[9,16]$.

Fiber bundle models have also been adopted to study the failure of glued interfaces of solid blocks [17-20]. Such interfaces as a part of complex constructions are assumed to sustain various types of external loads. In fiber-reinforced composites, where fibers are embedded in a matrix material,

*Electronic address: raischel@ica1.uni-stuttgart.de the fabrication of the fiber-matrix interface strongly affects the mechanical performance of the composite. Very recently we have shown that under shear loading of glued solid blocks, the interface elements may suffer not only stretching but also bending [21]. In order to capture this effect we proposed to discretize the interface in terms of beams which can be elongated and bent and break due to both deformation modes in a complex way. During the gradual failure of interfaces of solid blocks under shear, damaged regions of the interface can still transmit load contributing to the overall load bearing capacity of the interface. This can occur, for instance, when the two solids remain in contact at the failed regions and exert friction force on each other. In many applications the glue between the two interfaces has disordered properties but its failure characteristics are not perfectly brittle; the glue under shear may also yield carrying a constant load above the yield point.

We present an extension of models of the shear failure of glued interfaces considering that surface elements after failure still can have a certain load-bearing capacity. The disordered interface is represented by a parallel set of fibers with random breaking thresholds and linearly elastic behavior until failure. The broken fibers are assumed to carry a constant load which is a fraction $0 \leqslant \alpha \leqslant 1$ of their failure load. Varying the value of $\alpha$ the model interpolates between the perfectly brittle $(\alpha=0)$ and perfectly plastic $(\alpha=1)$ constitutive behavior of fibers. Based on analytic calculations and computer simulations, we show that the finite load-bearing capacity of failed fibers has a substantial effect on both the macroscopic response and microscopic damage process of the fiber bundle. When the load redistribution following fiber failure is short ranged, an interesting phase transition is revealed at a specific value of $\alpha$. 


\section{MODEL}

In order to model the shear failure of glued interfaces, we recently introduced a model [21] which represents the interface as an ensemble of parallel beams connecting the surfaces of two rigid blocks. The beams are assumed to have identical geometrical extensions (length $l$ and width $d$ ) and linearly elastic behavior characterized by the Young modulus $E$. In order to capture the failure of the interface, the beams are assumed to break when their deformation exceeds a certain threshold value. Under shear loading of the interface, beams suffer stretching and bending deformation, resulting in two modes of breaking. The stretching and bending deformation of beams can be expressed in terms of a single variable-i.e., longitudinal strain $\varepsilon=\Delta l / l$ - which enables us to map the interface model to the simpler fiber bundle models. The two breaking modes can be considered to be independent or combined in the form of a von Mises-type breaking criterion. The strength of beams is characterized by the two threshold values of stretching $\varepsilon_{1}$ and bending $\varepsilon_{2}$ a beam can withstand. The breaking thresholds are assumed to be randomly distributed variables of the joint probability distribution $p\left(\varepsilon_{1}, \varepsilon_{2}\right)$. The randomness of the breaking thresholds is supposed to represent the disorder of the interface material. After breaking of a beam the excess load has to be redistributed over the remaining intact elements. In Ref. [21] we presented a detailed study which demonstrated that the beam model of sheared interfaces with two breaking modes can be mapped into a simple fiber bundle model of a single breaking mode by an appropriate transformation of the fibers' strength disorder.

In the present paper, we extend our model by taking into account that failed surface elements can still carry some external load, increasing the load bearing capacity of the damaged interface. For simplicity, our study is restricted to discretizing the interface in terms of fibers which could then be further generalized to beams [21]. A bundle of parallel fibers is considered with breaking thresholds $\sigma_{t h}$ in the interval $0 \leqslant \sigma_{t h} \leqslant \sigma_{t h}^{\max }$ with a probability density $p\left(\sigma_{t h}\right)$ and distribution function $P\left(\sigma_{t h}\right)=\int_{0}^{\sigma_{t h}} p\left(\sigma_{t h}^{\prime}\right) d \sigma_{t h}^{\prime}$. We assume that after the breaking of a fiber at the failure threshold $\sigma_{t h}^{i}$, it may retain a fraction $0 \leqslant \alpha \leqslant 1$ of its ultimate load $\sigma_{t h}^{i}$; i.e., it will continue to transfer a constant load $\alpha \sigma_{\text {th }}^{i}$ between the surfaces. This assumption can be interpreted so that at the damaged areas of the interfaces the two solids still remain in contact, exerting, for instance, a friction force which may contribute to the overall load-bearing capacity. In many applications the glue between the two interfaces has disordered properties but its failure characteristics is not perfectly brittle; the glue under shear may also yield. The constitutive behavior of single fibers is illustrated in Fig. 1. Note that the load carried by the broken fibers is independent of the external load; furthermore, it is a random variable due to the randomness of the breaking thresholds. Varying the value of $\alpha$, the model interpolates between the perfectly brittle failure $(\alpha=0)$ and perfectly plastic $(\alpha=1)$ behavior of fibers. The load stored by the failed fibers reduces the load increment redistributed over the intact fibers, which strongly affects the process of gradual failure occurring under quasistatic loading of the interface. In

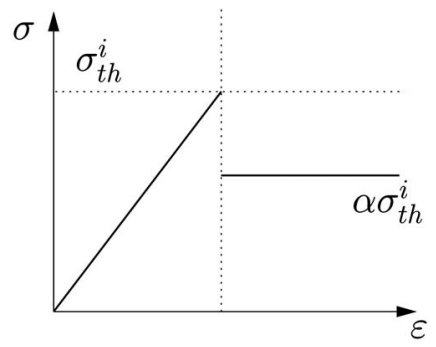

FIG. 1. Constitutive behavior of a single fiber: the fiber shows linearly elastic behavior up to the breaking threshold $\sigma_{t h}^{i}$; then, it keeps a fraction $0 \leqslant \alpha \leqslant 1$ of the ultimate load $\alpha \sigma_{t h}^{i}$.

the following we present a detailed study of the model system, varying the strength of plasticity $\alpha$. For the range of load sharing the two limiting cases of global and local load redistributions will be considered after failure events.

\section{TRANSITION TO PERFECT PLASTICITY}

Assuming global load sharing (GLS) after fiber breaking, the constitutive equation of the interface can be cast into a closed form. At an externally imposed deformation $\varepsilon$ the interface is a mixture of intact and broken fibers, which both contribute to the load-bearing capacity of the interface. Since the broken fibers retain a fraction $\alpha$ of their failure load, at the instant of fiber breaking only the reduced load $(1-\alpha) \sigma_{\text {th }}^{i}$ is redistributed over the intact fibers. Since the fraction of fibers having breaking threshold in the interval $[\varepsilon, \varepsilon+d \varepsilon]$ can be obtained as $p(\varepsilon) d \varepsilon$, the constitutive equation $\sigma(\varepsilon)$ reads as

$$
\sigma(\varepsilon)=\underbrace{E \varepsilon[1-P(\varepsilon)]}_{\sigma_{\mathrm{DFBM}}}+\underbrace{\alpha \int_{0}^{\varepsilon} E \varepsilon^{\prime} p\left(\varepsilon^{\prime}\right) d \varepsilon^{\prime}}_{\sigma_{\mathrm{Pl}}},
$$

where the integration is performed over the entire load history. The first term labeled $\sigma_{\mathrm{DFBM}}$ provides the load carried by the intact fibers, which corresponds to the classical dry fiber bundle model (DFBM) behavior $[3,8,22]$. The constitutive law of DFBM's is recovered in the limiting case $\alpha=0$, when the complete load of the failed fiber is transferred to the remaining intact fibers of the bundle. In the second term $\sigma_{P l}$, which accounts for the load carried by the broken fibers, the integral is calculated over the entire load history of the interface up to the macroscopic deformation $\varepsilon$. It can be seen in Eq. (1) that the value of $\alpha$ controls the relative importance of the elastic and plastic terms influencing the macroscopic response $\sigma(\varepsilon)$ and also the microscopic damage process of the system. When $\alpha$ is increased, less load is transferred to the intact fiber and in the limiting case $\alpha=1$ failed fibers retain their entire load, so no load transfer occurs. In this report, we explore the influence of the parameter $\alpha$ when it is tuned between these two extremal cases. In the following calculations the value of the fibers' Young modulus was set to unity $E=1$.

We note that the plastic fiber bundle model resembles up to some extent to the continuous damage fiber bundle model (CDFBM) worked out in Refs. [11,23]. The main assumption 
of the CDFBM is that due to the activation of certain internal degrees of freedom, the fibers undergo a gradual softening process reducing their Young modulus in consecutive partial failure events. The fibers always remain linearly elastic but with a Young modulus $E(k)=a^{k} E$, where the multiplication factor $0 \leqslant \alpha \leqslant 1$ describes the stiffness reduction in a single failure event and $k$ denotes the number of failures occurred. If the fibers can fail only once $(k=1)$ and keep their stiffness value constant, the constitutive law of the system reads as

$$
\sigma(\varepsilon)=E \varepsilon[1-P(\varepsilon)]+a E \varepsilon P(\varepsilon) .
$$

It was demonstrated in Refs. $[11,23]$ that increasing the number of times $k$ the fibers can fail, the CDFBM develops a plastic plateau, however with a mechanism completely different from the one considered here.

It is instructive to consider two fundamentally different cases of disorder distributions $P(\varepsilon)$-namely, bounded and unbounded ones, where the largest breaking threshold $\sigma_{t h}^{\max }$ takes a finite value or goes to infinity, respectively. In this report, we focus on two specific realizations; i.e., a uniform distribution between 0 and $\sigma_{t h}^{\max }$

$$
P\left(\sigma_{t h}\right)=\frac{\sigma_{t h}}{\sigma_{t h}^{\max }}, \quad 0 \leqslant \sigma_{t h} \leqslant \sigma_{t h}^{\max },
$$

and distributions of the Weibull type

$$
P\left(\sigma_{t h}\right)=1-e^{-\left(\sigma_{t h} / \lambda\right)^{m}}
$$

are considered where $\lambda$ and $m$ denote the characteristics strength and Weibull modulus of the distribution, respectively. For our study the Weibull distribution has the advantage that the amount of disorder in the failure thresholds can easily be controlled by the value of $m$.

The functional form of the constitutive behavior $\sigma(\varepsilon)$ is shown in Fig. 2 for both disorder distributions, Eqs. (3) and (4). It is interesting to note that for $\alpha<1$ there always exists a maximum of $\sigma(\varepsilon)$, just as in the case of the DFBM. Understress-controlled loading conditions, macroscopic failure occurs at the maximum of $\sigma(\varepsilon)$ so that the position and value of the maximum define the critical stress $\sigma_{c}$ and strain $\varepsilon_{c}$ of the bundle, respectively. It can be observed in Fig. 2 that the value of $\sigma_{c}$ and $\varepsilon_{c}$ are both higher than the corresponding values of DFBM, indicating that the presence of plastic fibers increases the macroscopic strength of the bundle. The decreasing part and the plateau of $\sigma(\varepsilon)$ can be realized under strain-controlled loading conditions gradually increasing $\varepsilon$. Under strain control the local load on the fibers is determined by the externally imposed deformation so that there is no load redistribution after fiber failure. The fibers break one by one in the increasing order of their failure thresholds $\sigma_{t h}^{i}=E \varepsilon_{t h}^{i}$. When the deformation $\varepsilon$ approaches the maximum value of the breaking thresholds $\varepsilon_{t h}^{\max }=\sigma_{t h}^{\max } / E$, all fibers must fail gradually so that the load of intact fibers $\sigma_{\mathrm{DFBM}}$ tends to zero, while that of the broken fibers $\sigma_{\mathrm{Pl}}$ takes a finite asymptotic value
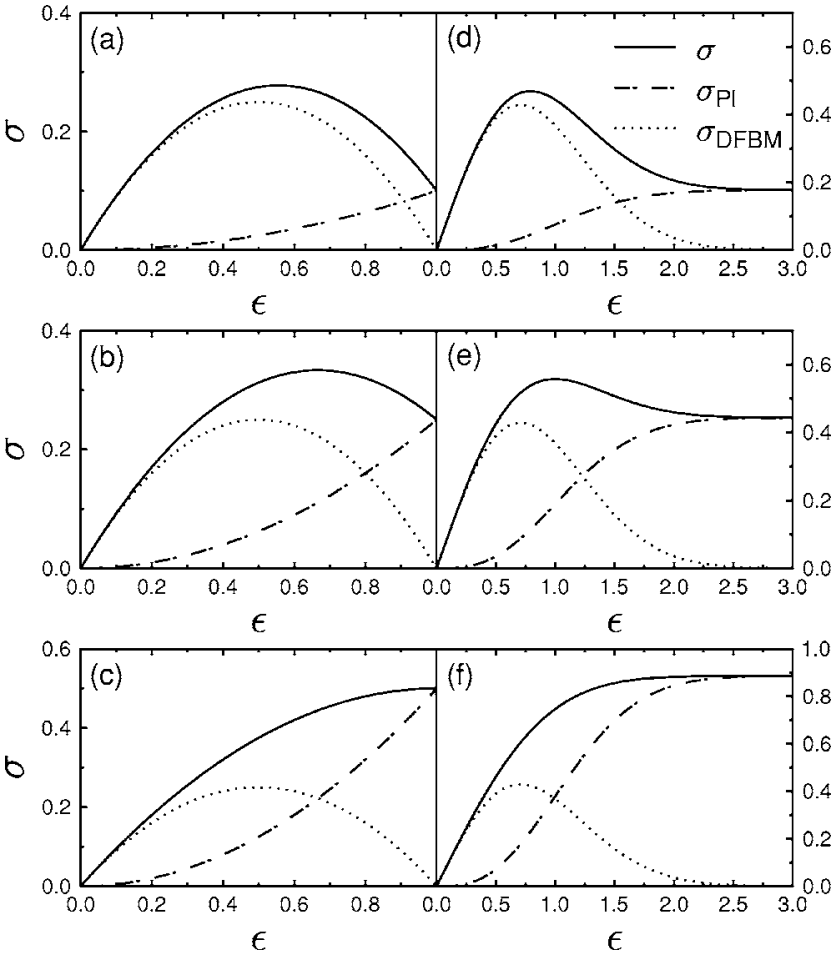

FIG. 2. Constitutive behavior $\sigma(\varepsilon)$ of the plastic fiber bundle for uniform (a), (b), (c) and Weibull distribution with $m=2$ (d), (e), (f) at $\alpha=0.2$ (a), (d), $\alpha=0.5$ (b), (e), and $\alpha=1.0$ (c), (f). The contributions of intact $\sigma_{\mathrm{DFBM}}$ and failed fibers $\sigma_{\mathrm{Pl}}$ are also shown. Note that $\sigma_{\text {DFBM }}$ is identical with the constitutive curve of simple dry fiber bundle models.

$$
\sigma_{\mathrm{Pl}} \rightarrow \widetilde{\sigma}=\alpha E \int_{0}^{\infty} \varepsilon^{\prime} p\left(\varepsilon^{\prime}\right) d \varepsilon^{\prime}=\alpha\left\langle\sigma_{t h}\right\rangle,
$$

where the integral is equal to the average fiber strength $\left\langle\sigma_{t h}\right\rangle$. When the strength of plasticity $\alpha$ is increased, the critical strain $\varepsilon_{c}$ and stress $\sigma_{c}$ and furthermore, the asymptotic stress of the plateau $\widetilde{\sigma}$ increase. The value of the critical deformation $\varepsilon_{c}$ can be obtained by differentiating Eq. (1) with respect to $\varepsilon$ and calculating the root [22]

$$
1-P\left(\varepsilon_{c}\right)-\varepsilon_{c} p\left(\varepsilon_{c}\right)[1-\alpha]=0,
$$

from which the critical stress follows as $\sigma_{c}=\sigma\left(\varepsilon_{c}\right)$. Equation (6) implies that in the limiting case of $\alpha \rightarrow 1$ the critical strain $\varepsilon_{c}$ tends to the maximum of the breaking thresholds $\varepsilon_{t h}^{\max }$, where $P\left(\varepsilon_{t h}^{\max }\right)=1$. For the uniform distribution, Eq. (3), we obtain

$$
\varepsilon_{c}=\frac{\varepsilon_{c}^{0}}{1-\alpha / 2}, \quad \text { hence } \varepsilon_{c} \rightarrow 2 \varepsilon_{\alpha \rightarrow 1}^{0}=\varepsilon_{t h}^{\max } .
$$

Here $\varepsilon_{c}^{0}$ denotes the critical strain of the DFBM $\varepsilon_{c}^{0}=\varepsilon_{t h}^{\max } / 2$, which can be obtained by setting $\alpha=0$ in Eq. (6). It follows that for unbounded threshold distributions like the Weibull distribution, $\varepsilon_{c}$ diverges so that perfect plasticity is only reached in the limit $\varepsilon_{c} \rightarrow \infty$. The functional form of the divergence is not universal, and due to the structure of the third term on the left-hand side of Eq. (6), $\varepsilon_{c}$ depends on the 


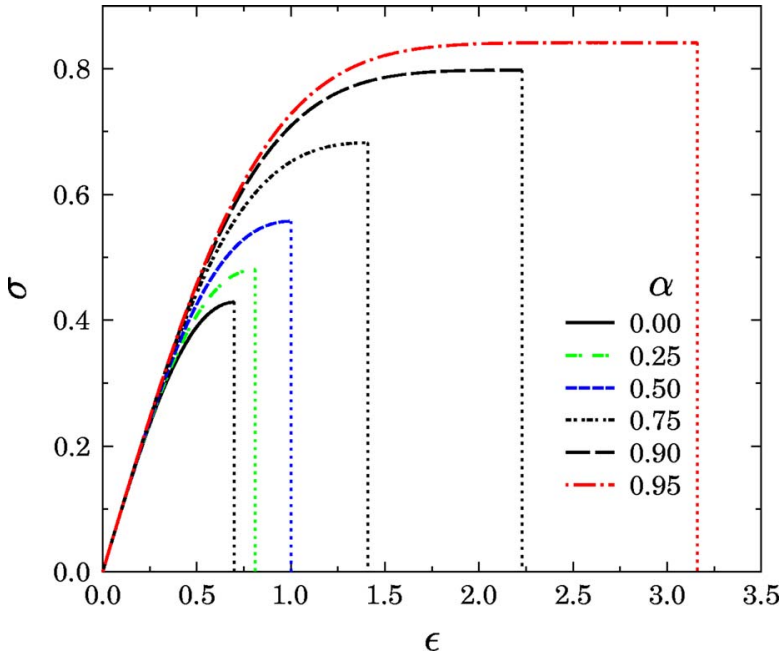

FIG. 3. (Color online) Simulations of stress controlled loading of a bundle of $N=1.6 \times 10^{6}$ fibers with Weibull distributed breaking thresholds $(\lambda=1, m=2)$. For clarity, the occurrence of macroscopic failure is indicated by vertical lines. Increasing $\alpha$ the constitutive behavior becomes perfectly plastic.

specific form of $p(\varepsilon)$. For the Weibull distribution, $\varepsilon_{c}$ as a function of $\alpha$ reads as

$$
\varepsilon_{c}=\varepsilon_{c}^{0}(1-\alpha)^{-1 / m}, \quad \text { where } \varepsilon_{c}^{0}=\lambda\left(\frac{1}{m}\right)^{1 / m},
$$

for any Weibull exponent $m$. Parallel to this, the decreasing part and the plateau of the constitutive curve $\sigma(\varepsilon)$ disappear so that $\sigma_{c}$ and $\widetilde{\sigma}$ converge to the same finite value, which is the average fiber strength $\left\langle\sigma_{t h}\right\rangle$ :

$$
\widetilde{\sigma} \rightarrow\left\langle\sigma_{t h}\right\rangle \text { and } \quad \sigma_{c} \rightarrow\left\langle\sigma_{t h}\right\rangle .
$$

The average fiber strength $\left\langle\sigma_{t h}\right\rangle$ can be determined as

$$
\left\langle\sigma_{t h}\right\rangle=\frac{\sigma_{t h}^{\max }}{2} \quad \text { and } \quad\left\langle\sigma_{t h}\right\rangle=\frac{1}{m} \Gamma\left(\frac{1}{m}\right)
$$

for the uniform and Weibull distributions, respectively. Here $\Gamma$ denotes the gamma function.

In order to illustrate this behavior, Fig. 3 presents constitutive curves for Weibull distributed fiber strength obtained by computer simulations of stress-controlled loading up to the critical point with $\lambda=1$ and $m=2$. It is apparent that in the limiting case of $\alpha \rightarrow 1$ the constitutive curve $\sigma(\varepsilon)$ reaches a plateau, indicating a perfectly plastic macroscopic state of the system. The position of the maximum $\varepsilon_{c}$ of the constitutive curves-i.e., the ending point of the curves - rapidly increases as $\alpha$ approaches 1, while the value of the maximum $\sigma_{c}$ tends to a finite value. In agreement with the analytic predictions, Eq. (8), simulations confirmed that $\varepsilon_{c}$ diverges as a power law whose exponent depends on the parameters of the strength distribution (see Fig. 4).

Controlling the external stress, the constitutive curve of the system, Fig. 3, can only be realized up to the maximum, since at the critical load $\sigma_{c}$ abrupt failure of the bundle occurs, breaking all the surviving intact fibers in a large burst. The fraction $\phi$ of fibers which break in the final burst caus-
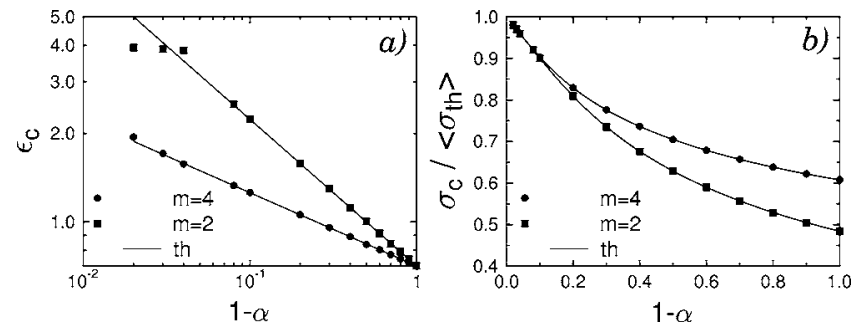

FIG. 4. Critical strain $\varepsilon_{c}$ (a) and critical stress $\sigma_{c}$ (b) as a function of $1-\alpha$ for a Weibull distribution with $\lambda=1, m=2$. Symbols: simulation results. Solid lines: analytical expressions Eqs. (8)-(10).

ing global failure can be determined as $\phi=1-P\left(\varepsilon_{c}(\alpha)\right)$, which is illustrated in Fig. 5 as a function of $1-\alpha$ for the specific case of a Weibull distribution

$$
\phi(\alpha)=e^{-1 / m(1-\alpha)} .
$$

It can be observed that as the system approaches the state of perfect plasticity $\alpha \rightarrow 1, \phi$ tends to zero. This demonstrates that more and more fibers break before global failure occurs, and perfect plasticity is obtained when the strongest fiber fails at the maximum of $\sigma(\varepsilon)$ (compare also to Fig. 3). This argument also implies that for $\alpha \rightarrow 1$, the difference of the microscopic damage process under stress- and straincontrolled loading disappears, the fibers break one by one without triggering avalanches of breakings.

\section{AVALANCHES OF FIBER BREAKINGS}

Under stress-controlled loading of the fiber bundle, the load dropped by a breaking fiber is redistributed over the intact ones. This load increment can give rise to further breakings which then may trigger an entire avalanche of failure events. The distribution $D(\Delta)$ of avalanche sizes $\Delta$ is an important quantity for the dynamical description of the loaded system. For the case of classical DFBM's $(\alpha=0)$ un-

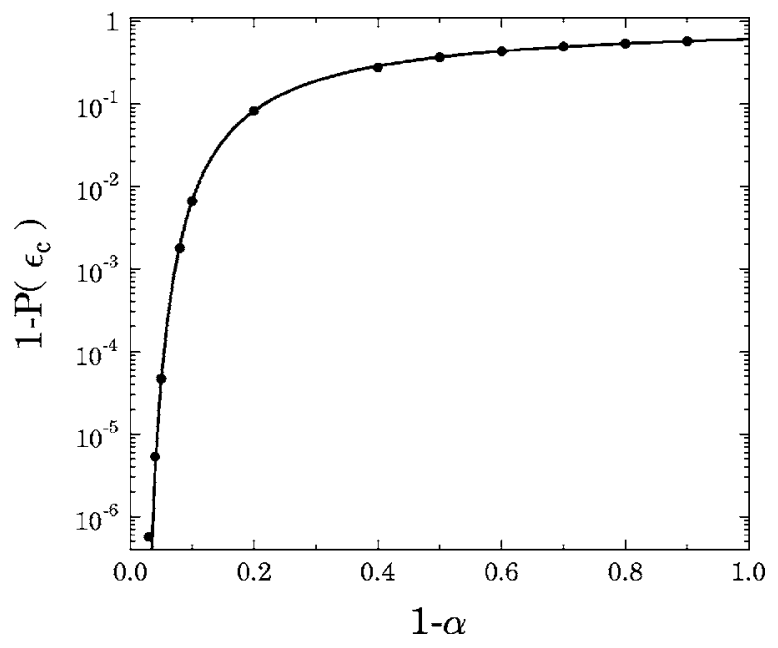

FIG. 5. Fraction of intact fibers $\phi=1-P\left(\varepsilon_{c}(\alpha)\right)$ at the point of macroscopic failure $\varepsilon_{c}$ vs $1-\alpha$ for a Weibull distribution of $\lambda=1$, $m=2$. Circles: GLS simulation results. Solid line: analytical solution, Eq. (11). 
der GLS conditions the avalanche size distribution $D(\Delta)$ can be obtained analytically $[8,24]$ as an integral, from which the asymptotic form of the distribution for large avalanches proved to be a power law

$$
D(\Delta) \propto \Delta^{-5 / 2}, \quad \Delta \rightarrow \infty .
$$

The value of the exponent $5 / 2$ is universal; it does not depend on the details of the disorder distribution of the failure thresholds [8,24].

In order to obtain the analytical solution for the avalanche distribution in the presence of plastic fibers $\alpha \neq 0$, we can follow the derivation of Refs. [8,24], taking into account that the average number of fibers, $a(\varepsilon, \alpha) d \varepsilon$, which break as a consequence of the load increment caused by a fiber breaking at the deformation $\varepsilon$ is reduced by a factor of $(1-\alpha)$ :

$$
a(\varepsilon, \alpha) d \varepsilon=\frac{\varepsilon p(\varepsilon)(1-\alpha)}{1-P(\varepsilon)} d \varepsilon .
$$

Taking into account that the critical deformation $\varepsilon_{c}$ where macroscopic failure occurs also depends on $\alpha$, the avalanche size distribution $D(\Delta)$ can be cast in the form

$$
\frac{D(\Delta)}{N}=\frac{\Delta^{\Delta-1}}{\Delta !} \int_{0}^{\varepsilon_{c}(\alpha)} a(\varepsilon, \alpha)^{\Delta-1} e^{-a(\varepsilon, \alpha) \Delta}[1-a(\varepsilon, \alpha)] p(\varepsilon) d \varepsilon .
$$

For the specific case of the Weibull distribution with an arbitrary modulus $m$ the general equation (14) can be written in the form

$$
D(\Delta, \alpha)=\frac{\Delta^{\Delta-1}}{\Delta ![m(1-\alpha)]^{2} \Delta_{c}^{\Delta+1}}\left[\gamma\left(\Delta, \Delta_{c}\right)+\Delta_{c}^{\Delta} m(1-\alpha) e^{-\Delta_{c}}\right],
$$

where $\Delta_{c}$ depends on the amount of disorder $m$ and on the strength of plasticity $\alpha$ :

$$
\Delta_{c}=\Delta+\frac{1}{m(1-\alpha)} .
$$

In Eq. (15), $\gamma$ denotes the incomplete gamma function. ${ }^{1}$ Two limiting cases can be distinguished in the solution: first, for $\alpha \rightarrow 0$ the classical power-law dependence, Eq. (12), is recovered. This analytic solution is illustrated in Fig. 6 for a Weibull distribution with $m=2$, where a power law of $D(\Delta)$ is apparent for $\alpha<0.9$. However, for the limiting case of $\alpha \rightarrow 1$, we have to consider the behavior of the argument $\Delta_{c}$ of the analytic solution, Eq. (15). For $\alpha \approx 1$, there will be a regime of $\Delta$ values where the term $1 /[m(1-\alpha)]$ dominates over $\Delta$, resulting in a faster decay of the distribution $D(\Delta)$ than any power. Still, for any values of $\alpha$ in the limiting case $\Delta \gg \Delta_{c}(\alpha)$, the usual mean-field power-law behavior, Eq. (12), is asymptotically recovered. Avalanche-size distributions $D(\Delta)$ obtained from computer simulations at various different values of $\alpha$ are presented in Fig. 7. In good quantitative agreement with the analytic predictions, the numeri-

\footnotetext{
${ }^{1}$ There are several definitions of the incomplete gamma function, we use $\gamma(a, x)=\int_{0}^{x} e^{-t} t^{a-1} d t$
}

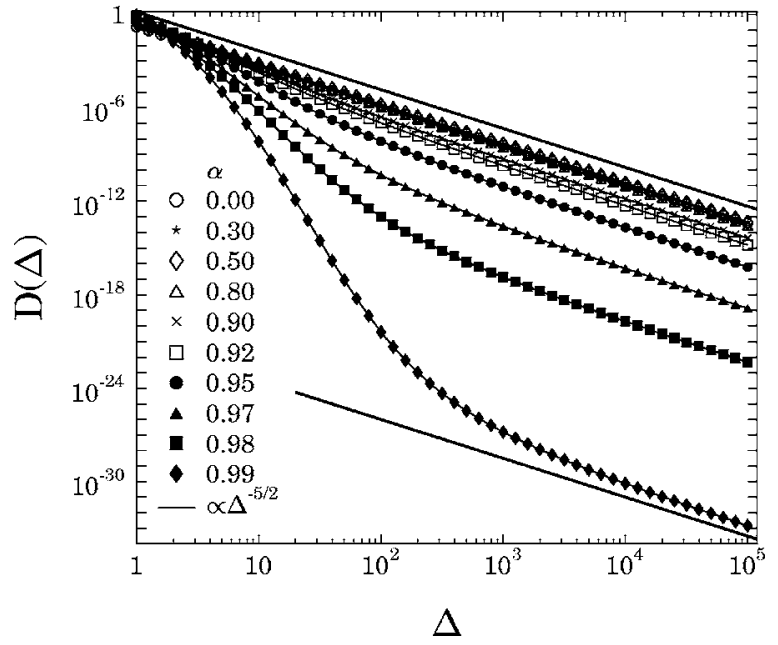

FIG. 6. Analytic solution of the avalanche size distribution $D(\Delta)$ at various different values of $\alpha$. For $\alpha \rightarrow 0$ the usual power-law distribution is recovered, whereas for $\alpha \rightarrow 1$ an exponential decay of $D(\Delta)$ is obtained. For the specific calculations a Weibull distribution was used with $m=2$.

cal results can be well fitted by a power law of exponent $5 / 2$ for moderate values of $\alpha$. However, for $\alpha>0.9$ strong deviations from the power law, Eq. (12), can be observed for intermediate avalanche sizes $1 \leqslant \Delta \leqslant 10^{3}$, which appears to be an exponential decay. Although in the analytical solution the asymptotic power-law behavior is still visible for very large $\Delta$ (see Fig. 6), computer simulations in Fig. 7 show solely a very steep decrease. It can be seen in the analytic solution in Fig. 6 that the relative frequency of avalanches of size $\Delta>O\left(10^{3}\right)$ is $D=O\left(10^{-30}\right)$ for $\alpha=0.99$, so it would require extremely large systems to count any such events. The size of the largest avalanche $\Delta_{\max }$ is plotted in Fig. 8 as a function of $\alpha$. Obviously, $\Delta_{\max }$ is a monotonically decreasing function of $\alpha$ whose decrease gets faster in the regime where

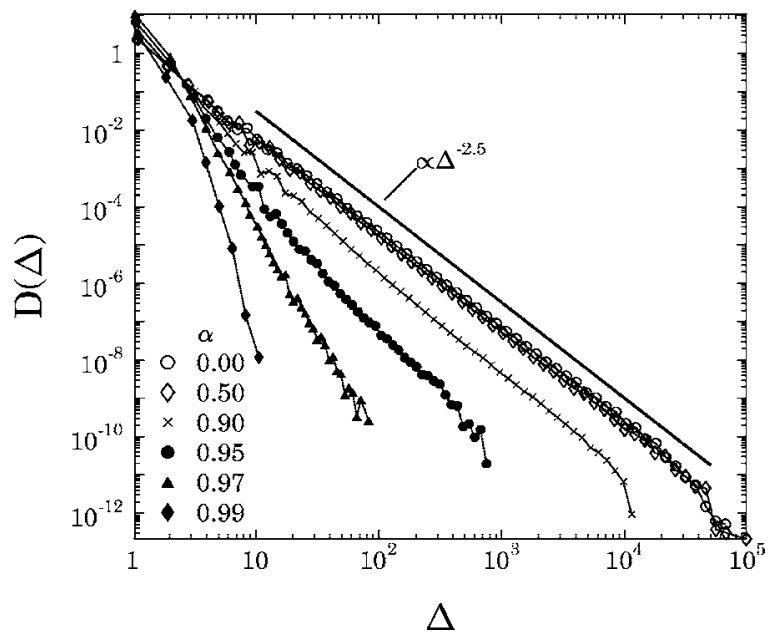

FIG. 7. Distribution $D(\Delta)$ of avalanches of size $\Delta$ for various values of $\alpha$ obtained by computer simulations for a system of $N=1.6 \times 10^{7}$ fibers with Weibull-distributed failure thresholds $m$ $=2$. Satisfactory agreement is obtained with the analytic results presented in Fig. 6. 


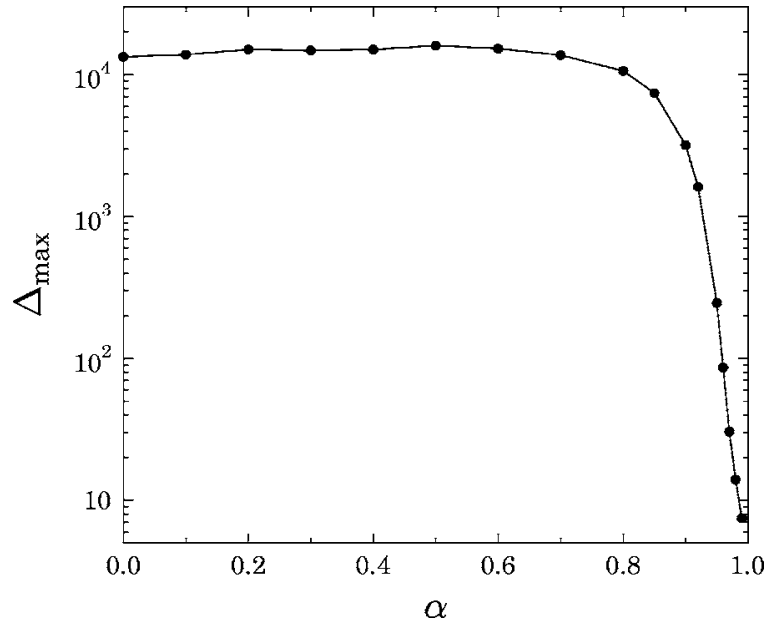

FIG. 8. Size of the largest avalanche in a GLS simulation with a Weibull distribution of $m=2$.

the distribution $D(\Delta)$ exhibits the crossover to the fasterdecaying form.

An important consequence of the analytic solution, Eqs. (15) and (16), is that the characteristic avalanche size where the crossover occurs from a power law to a faster-decaying exponential form also depends on the amount of disorder; i.e., the stronger the disorder is, the larger the crossover size gets at a given $\alpha$.

\section{LOCAL LOAD SHARING}

From experimental and theoretical point of view, it is very important to study the behavior of the plastic bundle when the interaction of fibers is localized. In the case of local load sharing (LLS) under stress-controlled external loading conditions, the load dropped by the broken fiber is redistributed in a local neighborhood of the fiber, giving rise to high stress concentration in the vicinity of failed regions. Stress concentration leads to correlated growth of clusters of broken fibers (cracks), which plays a crucial role in the final breakdown of the system; i.e., macroscopic failure of the bundle occurs due to the instability of a broken cluster which then triggers an avalanche of failure events where all the remaining intact fibers break. This effect typically leads to a more brittle constitutive behavior of the system and the appearance of nontrivial spatial and temporal correlations in the damage process $[17,23,25,26]$.

In the plastic bundle, after a fiber breaks it still retains a fraction $\alpha$ of its failure load $\sigma_{t h}$ so that only the amount $(1-\alpha) \sigma_{t h}$ is redistributed over the intact fibers in the neighborhood. It implies that the load-bearing broken fibers reduce the stress concentration around failed regions, giving rise to stabilization which also affects the temporal and spatial evolution of damage during the loading process.

In the following we consider a bundle of $N$ fibers organized on a square lattice of size $L \times L$ with periodic boundary conditions. The fibers are assumed to have Weibulldistributed strength, Eq. (4), where the value of $\lambda$ is always set to unity and for the Weibull modulus two different values

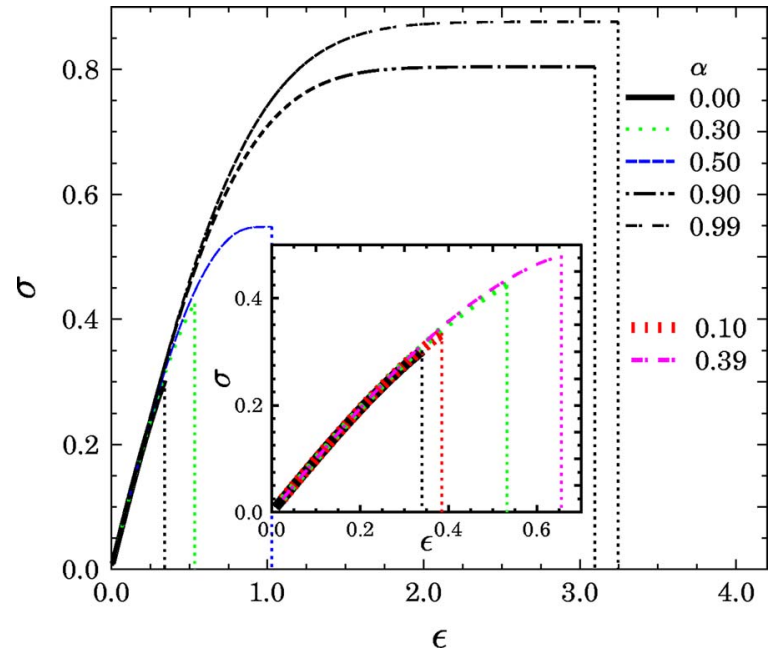

FIG. 9. (Color online) Constitutive law $\sigma(\varepsilon)$ of the LLS bundle obtained by computer simulations of a system of size $L=401$ for several different values of $\alpha$. The inset shows a magnified view of $\sigma(\varepsilon)$ for the regime $\alpha<0.4$. For clarity, vertical lines indicate the location of macroscopic failure. For the breaking thresholds a Weibull distribution was used with $m=2$.

are considered: $m=2$ (large disorder) and $m=4$ (smaller disorder). After a failure event the load dropped by the broken fiber $(1-\alpha) \sigma_{\text {th }}^{i}$ is equally redistributed over the nearest and next-nearest intact neighbors in the square lattice; i.e., the local neighborhood of a broken fiber contains at most eight intact sites. Stress-controlled simulations have been carried out for system sizes ranging from $L=33$ to $L=801$, varying the strength of plasticity $0 \leqslant \alpha \leqslant 1$.

\section{A. Macroscopic response}

It has been shown for DFBM's where broken fibers carry no load, that the macroscopic response of the bundle when the interaction of fibers is localized follows the constitutive law of the corresponding GLS system with a reduced critical strain and stress; i.e., the LLS bundle behaves macroscopically in a more brittle way than its GLS counterpart $[23,25,26]$. Figure 9 shows the constitutive curve of a plastic bundle of size $L=401$ for several different values of $\alpha$. It can be observed that for $\alpha \approx 0$ the constitutive curve exhibits the usual LLS behavior; i.e., the macroscopic failure is preceded by a relatively short nonlinear regime and global failure occurs in an abrupt manner. The position of the macroscopic failure defines the value of the critical strain $\varepsilon_{c}^{L L S}$ and stress $\sigma_{c}^{L L S}$. It is very interesting to note that when $\alpha$ is increased, the LLS constitutive curves practically recover the behavior of the corresponding GLS system; i.e., for $\alpha \geqslant 0.4$ the macroscopic failure occurs when reaching the plateau of $\sigma(\varepsilon)$.

The convergence of the LLS system to the GLS macroscopic behavior is better seen in Fig. 10 where the relative difference of the critical stresses $\sigma_{c}^{G L S}(\alpha)$ and $\sigma_{c}^{L L S}(\alpha)$ of the global and local load-sharing bundles is presented. It can be seen in the figure that there exists a threshold value $\alpha_{c}$ of $\alpha$ above which the macroscopic response of the LLS bundle becomes very close to the corresponding GLS system, while 


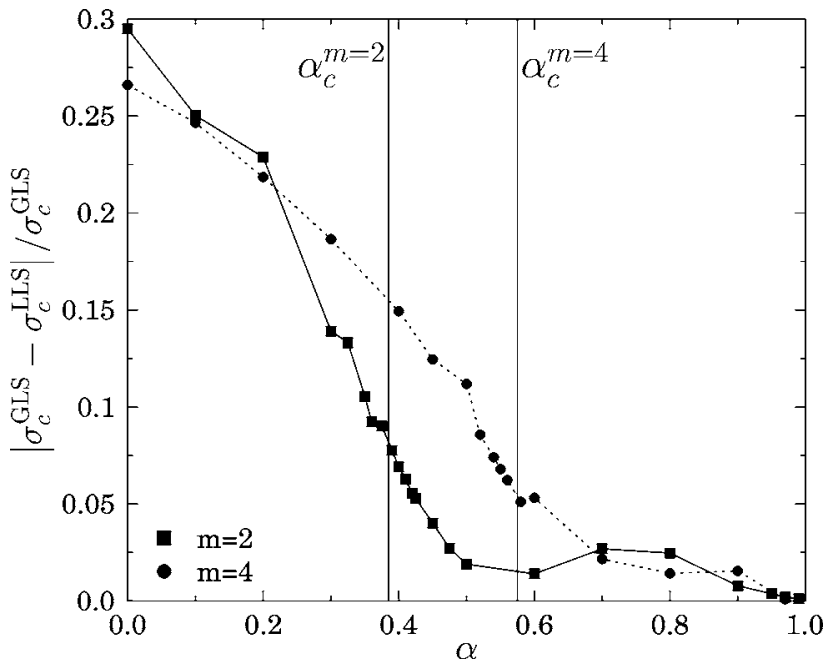

FIG. 10. The relative difference of the critical stresses $\sigma_{c}^{G L S}$ and $\sigma_{c}^{L L S}$ of global and local load-sharing systems as a function of $\alpha$ for two different values of the Weibull modulus $m$. The vertical lines indicate the critical values of $\alpha$, which were obtained in Sec. V C.

below $\alpha_{c}$ the constitutive behavior of the bundle changes continuously from the usual LLS response with a high degree of brittleness $(\alpha=0)$ to the global load sharing behavior. It seems that at $\alpha_{c}$ a continuous transition occurs between the two regimes. The transition indicates that as a consequence of the reduction of stress concentration around failed fibers, the bundle can sustain higher external loads and is able to keep its integrity until the maximum of $\sigma(\varepsilon)$ is reached.

\section{B. Bursts of fiber breakings}

The evolution of the macroscopic response of the system with increasing $\alpha$ is accompanied by interesting changes of the damage process on the microlevel, characterized by avalanches of fiber breakings and the cluster structure of failed regions. The avalanche statistics presented in Fig. 11 shows remarkable features. For $\alpha \approx 0$, due to the high stress concentration around failed fibers, the LLS bundle can only tolerate small avalanches so that the avalanche-size distribution $D(\Delta)$ decays rapidly. With increasing $\alpha$ the higher amount of load kept by broken fibers can stabilize the bundle even after larger bursts; hence, the cutoff of the distributions moves to higher values. It is interesting to note that also the functional form of the distribution $D(\Delta)$ changes; i.e., when $\alpha$ approaches $\alpha_{c}$ the exponential cutoff disappears and the distribution becomes a power law

$$
D(\Delta) \sim \Delta^{-\mu}
$$

for large avalanches. The exponent $\mu$ of the power law was determined numerically as $\mu^{L L S}=1.5 \pm 0.07$, which is significantly lower than the mean-field value $\mu^{G L S}=2.5$ [8]. Increasing $\alpha$ above the critical point an exponential cutoff occurs and the power-law regime of large avalanches gradually disappears. Comparing Fig. 11 to the corresponding GLS results presented in Fig. 7, it is apparent that above $\alpha_{c}$ the LLS distributions $D(\Delta)$ have the same functional form and follow the same tendency with increasing $\alpha$ as the mean-field

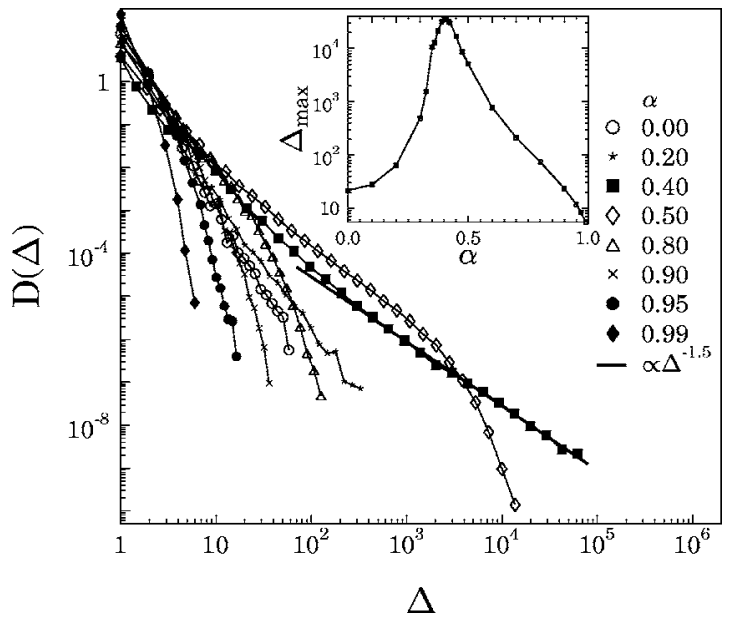

FIG. 11. Avalanche-size distributions $D(\Delta)$ obtained by computer simulations for the system size $L=401$ with local load sharing, using Weibull-distributed failure thresholds $m=2$. The powerlaw fit is demonstrated for $\alpha=0.4$. In the inset the largest avalanche $\Delta_{\max }$ is plotted versus $\alpha$.

results. It can be concluded that the avalanche statistics presents the same transitional behavior between the local loadsharing and mean-field regimes as observed for the macroscopic response. The same value of $\mu^{L L S}$ was obtained numerically for $m=4$, indicating the universality of the exponent with respect to the strength of disorder. The transition is more evident in the inset of Fig. 11, where the size of the largest avalanche $\Delta_{\max }$ is plotted as a function of $\alpha$. The sharp peak indicates the transition point whose position defines $\alpha_{c}$, while in GLS the largest avalanche $\Delta_{\max }$ was a monotonically decreasing smooth function (compare to Fig. 8).

\section{Spatial structure of damage}

Gradually increasing the external load in the fiber bundle, the weakest fibers break first in an uncorrelated manner. Since the load is redistributed solely over the intact neighbors of the broken fiber, the chance of fiber breakings increases in the vicinity of damage regions. This effect can result in correlated growth of clusters of broken fibers with a high stress concentration around their boundaries. The larger the cluster is, the higher stress concentration arises. Global failure of the bundle occurs when, due to an external load increment, one of the clusters becomes unstable and grows until all fibers break. The spatial structure of the damage emerging when the interaction of fibers is localized can be characterized by studying the statistics and structure of clusters of broken fibers. Former studies of the limiting case of very localized interactions have revealed that the size of the largest cluster in the system is rather limited; furthermore, it is independent of the system size. Since the clusters are relatively small, merging of neighboring clusters does not occur frequently. The clusters themselves are found to be compact objects dispersed homogeneously over the cross section of the bundle $[23,27,28]$.

In Fig. 12 the latest stable configuration of the bundle is presented just before catastrophic failure occurs at the critical 


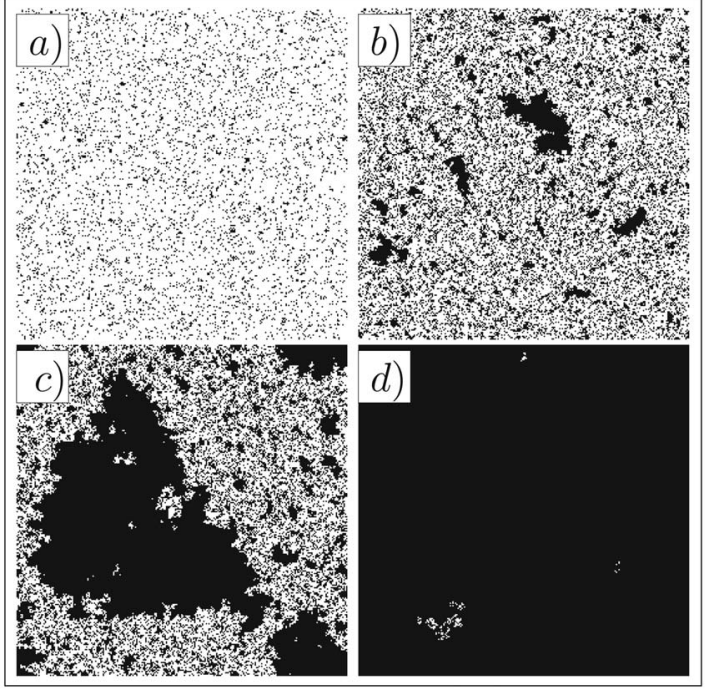

FIG. 12. Latest stable configuration in LLS simulations of a system of size $L=401$, with a Weibull strength distribution $m=2$ at different values of the control parameter $\alpha$ (a) 0.0 , (b) 0.35 , (c) 0.4 , and (d) 0.6. The strength of the largest cluster $P_{\infty}$ in the lattices are (a) 0.003, (b) 0.097, (c) 0.517, and (d) 0.999. Broken and intact fibers are indicated by black and white, respectively.

load $\sigma_{c}^{L L S}$ for several different values of $\alpha$. For $\alpha \approx 0$ we note only small clusters of broken fibers as is expected for LLS bundles [Fig. 12(a)]. With increasing $\alpha$, these clusters grow and adjacent clusters can even merge, further increasing the typical cluster size [Fig. 12(b)]. Around the critical value of $\alpha \approx 0.4$, a spanning cluster of broken fibers seems to appear [Fig. 12(c)], whereas for higher values of $\alpha>0.4$ almost all fibers have failed [Fig. 12(d)] already by the time the critical stress is reached. The existence of very large clusters is the direct consequence of the increased load-bearing capacity of broken fibers.

Clusters of broken fibers were identified in the square lattice using the Hoshen-Kopelman algorithm. We evaluated the distribution of cluster sizes $n(S)$ in the last stable configuration just before macroscopic failure occurs. The behavior of $n(S)$ shows again the transitional nature we have observed for other quantities. It can be seen again in Fig. 13 that a well-defined $\alpha_{c}$ exists which separates two regimes: for $\alpha<\alpha_{c}$ the clusters are small and $n(S)$ has a steep decrease. Approaching $\alpha_{c}$, the cluster size distribution $n(S)$ tends to a power law

$$
n(S) \sim S^{-\tau}
$$

where the value of the exponent was obtained as $\tau=2.35 \pm 0.08$ which is higher than the corresponding exponent of two-dimensional (2D) percolation on a square lattice $\tau=187 / 91 \approx 2.0549$ [29]. Note that in the regime where spanning clusters exist $(\alpha \geqslant 0.4)$, the distribution $n(S)$ contains only the finite clusters.

In order to characterize the evolution of the cluster structure when $\alpha$ is changed and to reveal the nature of the transition occurring at $\alpha_{c}$, we calculated the average cluster size

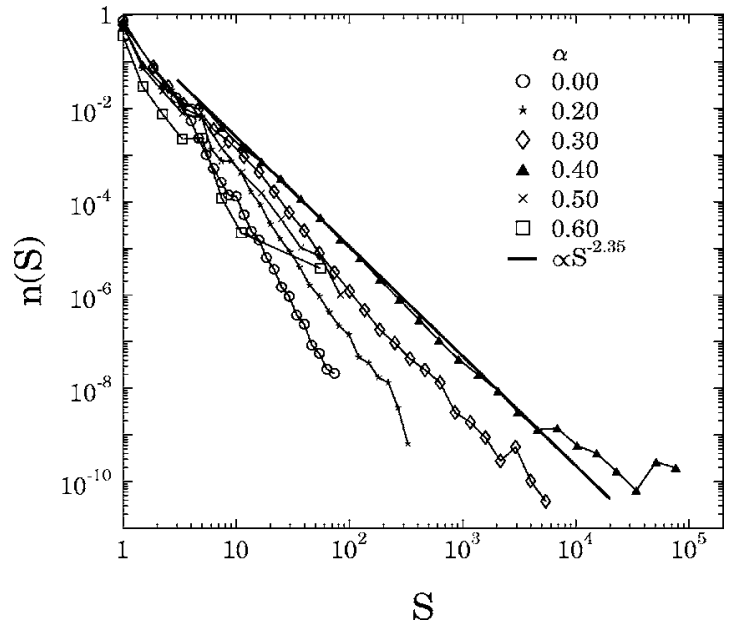

FIG. 13. Distribution $n$ of the size $S$ of broken clusters in LLS simulations with a Weibull distribution $m=2$, for different values of $\alpha$. The spanning clusters were excluded from the distributions for $\alpha \geqslant 0.4$.

$S_{a v}$ as the ratio of the second and first moments of the cluster size distribution

$$
S_{a v}=\frac{m_{2}}{m_{1}} .
$$

The $k$ th moment $m_{k}$ of the distribution $n(S)$ is defined as

$$
m_{k}=\sum_{S} S^{k} n(S)-S_{\text {max }}^{k},
$$

where the largest cluster is excluded from the summation. Figure 14 presents $S_{a v}$ as a function of $\alpha$ for different system sizes ranging from $L=33$ to $L=801$. It can be seen in the figure that for each value of $L$ the average cluster size $S_{a v}$ has a maximum at a well-defined value of $\alpha$, which becomes a sharp peak with increasing $L$; i.e., the peak becomes higher and narrower for larger systems. The observed behavior is typical for continuous phase transitions,

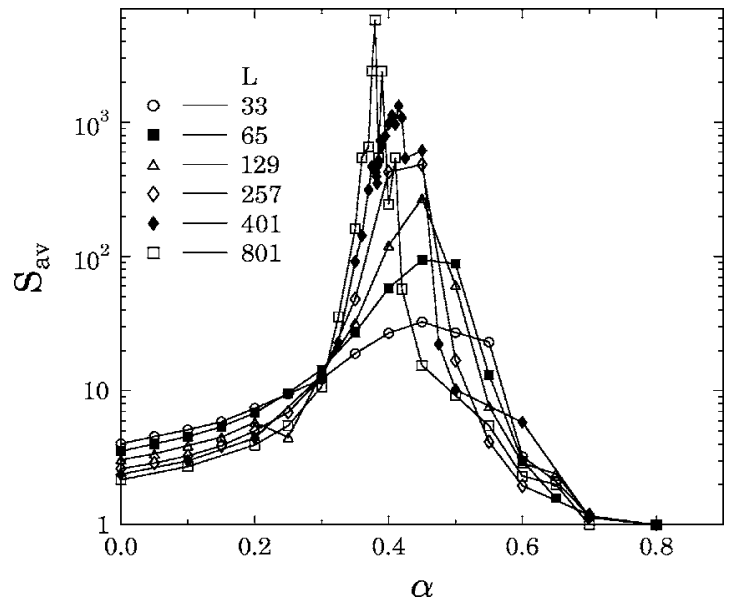

FIG. 14. Average cluster size $S_{a v}=m_{2} / m_{1}$ as a function of $\alpha$ for different system sizes $L$. Simulation results were obtained with a Weibull distribution $m=2$. 


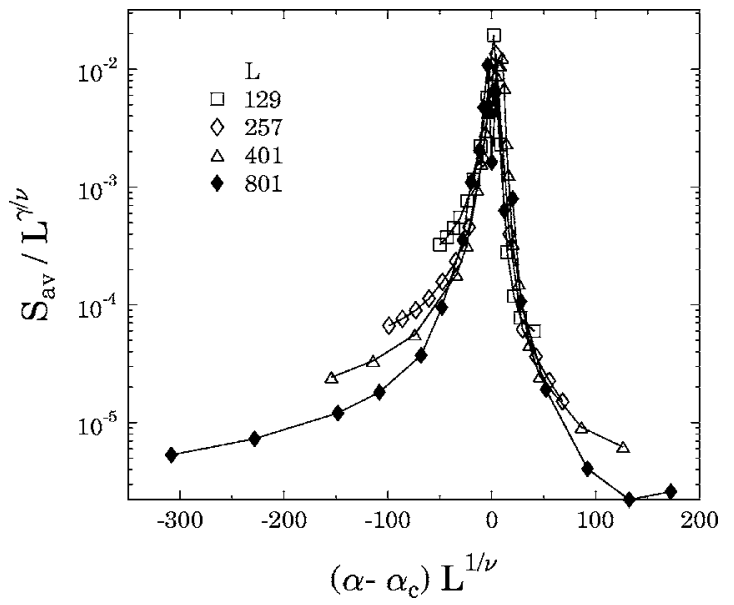

FIG. 15. Finite-size scaling of the average cluster size $S_{a v}$ presented in Fig. 14. The good quality collapse obtained enables us to determine the value of the critical exponents $\gamma$ and $\nu$ with a relatively good precision.

where the position of the maximum defines the critical point of the finite-size system. Based on the analogy to critical phenomena we tested the validity of the scaling law $S_{a v} \sim L^{\gamma / \nu} \phi\left[\left(\alpha-\alpha_{c}\right) L^{1 / \nu}\right]$, where $\phi$ denotes the scaling function of $S_{a v}[29,30]$. The results presented in Fig. 15 were obtained by varying the values of the critical point $\alpha_{c}$ and of the critical exponent of the susceptibility $\gamma$ and correlation length $\nu$ until the best data collapse was reached. It can be observed in Fig. 15 that in the vicinity of the critical point $\alpha_{c}$ a good quality data collapse is obtained using the values $\alpha_{c}=0.385 \pm 0.01, \gamma=2.0 \pm 0.15$, and $\nu=1.0 \pm 0.1$, where the critical exponents are only slightly different from the percolation exponents of $\gamma=43 / 18 \approx 2.389$ and $\nu=4 / 3 \approx 1.33$ in 2D [29].

At the critical point a spanning cluster of broken fibers occurs which is much larger than the other clusters. In order to characterize the strength of the spanning cluster we calculated the probability $P_{\infty}(\alpha)$ that a failed fiber belongs to the largest cluster. For percolation the quantity $P_{\infty}$ plays the role of the order parameter whose value distinguishes the phases of the system. Similarly to percolation lattices, we find numerically a sharp rise from $P_{\infty}=0$ to $P_{\infty}=1$ at $\alpha_{c} \approx 0.4$; see Fig. 16. When the system size $L$ is increased $P_{\infty}$ tends to a step function indicating that the transition becomes sharper. Assuming the scaling law $P_{\infty} \sim L^{-\beta / \nu} \psi\left[\left(\alpha-\alpha_{c}\right) L^{1 / \nu}\right]$ of the order parameter for finite-size systems, where $\psi$ denotes the scaling function and $\beta$ is the order parameter exponent $[29,30]$, we replotted the data in Fig. 17. The good quality of the data collapse was obtained with the parameter values $\alpha_{c}=0.33 \pm 0.01, \beta=0.15 \pm 0.06$, and $\nu=0.95 \pm 0.1$. Note that the value of $\nu$ agrees well with the one determined by the finite-size scaling of the average cluster size $S_{a v}$; larger deviations occur only for the critical point $\alpha_{c}$. The order parameter exponent $\beta$ is compatible with the percolation value $\beta=5 / 36 \approx 0.13$.

\section{Random crack nucleation versus crack growth}

The failure mechanism of disordered materials and its relation to the amount of disorder has long been discussed in

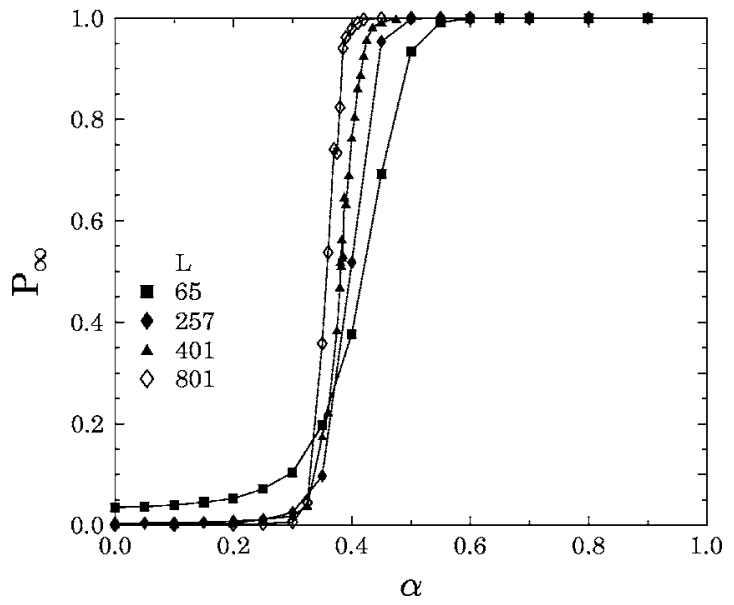

FIG. 16. Order parameter $P_{\infty}$ vs $\alpha$ for several system sizes $L$ with the Weibull index $m=2$.

the literature $[1,2,23,28,31-34]$. When the material has a low degree of disorder only a small amount (if any) of damage occurs prior to macroscopic failure. In this case even the nucleation of a single microcrack can lead to localization and abrupt failure of the system. Increasing the amount of disorder, the macroscopic failure is preceded by a larger and larger precursory activity; i.e., a large amount of damage accumulates and local breakings can trigger bursts of breaking events [8]. Since cracks nucleate randomly, the process of damage before localization resembles percolation up to some extent. Stress concentration builds up around failed regions which might lead to correlated growth of the nucleated cracks $[12,23,31,35]$. Increasing the strength of disorder, correlation effects become less dominating and in the limit of infinite disorder the damage accumulation process can be mapped to percolation [36].

We have shown above that in the plastic fiber bundle model (PFBM), the load-bearing capacity of broken fibers has a substantial effect on the process of failure when the load redistribution is localized due to the reduction of the

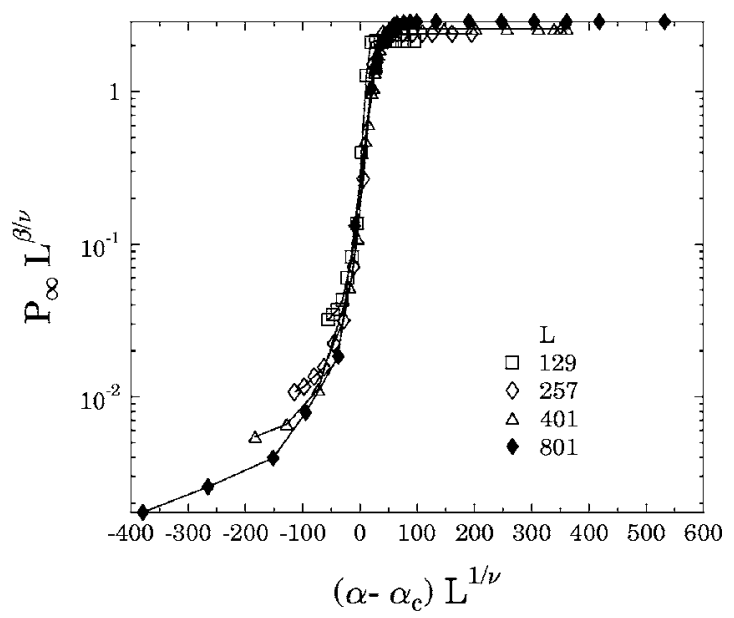

FIG. 17. Finite size scaling of the order parameter $P_{\infty}$ presented in Fig. 16. The parameter values used to obtain the best quality data collapse agree well with the ones determined by the finite size scaling of $S_{a v}$. 


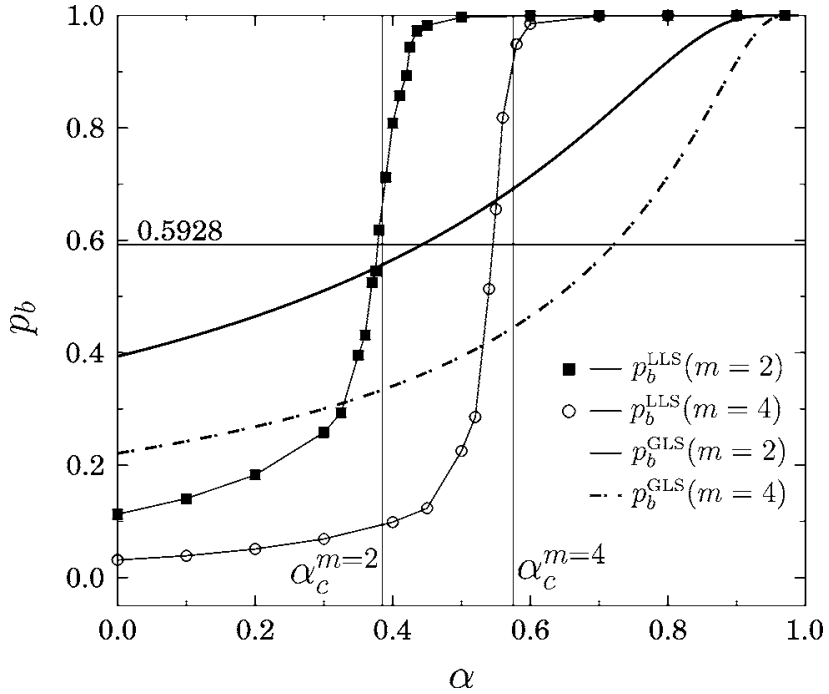

FIG. 18. The fraction of broken fibers $p_{b}$ at $\sigma_{c}$ as a function of $\alpha$ for fiber bundles of LLS and GLS with different strengths of disorder $m=2$ and $m=4$. The vertical line indicates the critical point obtained as the position of the maximum of the average cluster size (see Fig. 14). The critical probability of percolation $p_{c}$ on the square lattice is indicated by the horizontal line. Note that for both disorder distributions in LLS, the location where $p_{b}^{L L S}$ exceeds $p_{c}$ practically coincides with the corresponding critical point $\alpha_{c}$, indicating the percolation nature of the transition.

stress concentration along cracks. In order to give a quantitative characterization of damage accumulation in our model, we determined the fraction of broken fibers $p_{b}$ at global failure $\sigma_{c}$ as a function of the strength of plasticity $\alpha$. The quantity $p_{b}$ can also be interpreted as the probability $0 \leqslant p_{b} \leqslant 1$ that a randomly chosen fiber in the bundle is broken which makes it possible to compare the spatial structure of damage to percolation lattices [29] generated with the occupation probability $p=p_{b}[1,2,26,36]$. The results are presented in Fig. 18 for the system size $L=401$ and Weibull parameters $m=2$ and $m=4$ plotting also the corresponding GLS results for comparison. In the case of local load sharing, when the failure load of fibers is almost entirely redistributed locally $(\alpha \approx 0)$ only a small damage can accumulate up to global failure $p_{b}^{L L S} \approx 0.1-0.2$, keeping the integrity of the system. Comparing the curves of different Weibull indices $m$ it follows that the stronger the disorder is, the larger amount of damage the system can tolerate at the same value of $\alpha$. In the vicinity of the respective $\alpha_{c}$, the breaking fraction $p_{b}^{L L S}$ rapidly increases and converges to the maximum value $p_{b}^{L L S} \approx 1$, which implies that in the regime $\alpha>\alpha_{c}$ practically no localization occurs, and the bundle can remain stable until almost all fibers break.

It is instructive to compare this behavior to the case of GLS, where those fibers break up to the critical point whose breaking threshold falls below $\sigma_{c}$; hence, $p_{b}^{G L S}(\alpha)$ can simply be obtained as $p_{b}^{G L S}=P\left(\sigma_{c}(\alpha)\right)$. It can be seen in Fig. 18 that under global load sharing for $\alpha \approx 0$ a significantly larger fraction of fibers fails without destroying the system than in the LLS bundle. The breaking fraction $p_{b}$ is a monotonically increasing function of $\alpha$ irrespective of the range of load sharing; however, in the vicinity of the critical point of LLS
TABLE I. Summary of the critical exponents of the plastic fiber bundle model with local load sharing. For comparison the value of the corresponding critical exponents of percolation are also shown. For the perimeter fractal dimension $D_{p}$ of the PFBM a range is given.

\begin{tabular}{ccc}
\hline \hline Critical exponents & PFBM & Percolation \\
\hline$\beta$ & $0.15 \pm 0.06$ & $5 / 36 \approx 0.13$ \\
$\gamma$ & $2.0 \pm 0.15$ & $43 / 18 \approx 2.38$ \\
$\tau$ & $2.35 \pm 0.08$ & $187 / 91 \approx 2.05$ \\
$\nu$ & $1.0 \pm 0.1$ & $4 / 3 \approx 1.33$ \\
$D$ & 2.0 & $D=91 / 48 \approx 1.896$ \\
$D_{p}$ & $1.0-2.0$ & $7 / 4=1.75$ \\
$\mu$ (bursts) & $1.5 \pm 0.07$ & \\
\hline \hline
\end{tabular}

bundles $p_{b}^{L L S}$ exceeds the smoothly rising GLS curves $p_{b}^{G L S}$. Note that depending on the threshold distribution $P$ of fibers, even at $\alpha=0$ the value of $p_{b}^{G L S}$ can be smaller or larger than the critical percolation probability $p_{c}$ of the corresponding lattice type, since (contrary to fuse networks [31,34] or discrete element models [37]) fracture in fiber bundles is not related to the appearance of a spanning cluster of failed elements. Varying $\alpha$ as a control parameter, formally the GLS results could be perfectly mapped onto a percolation problem: at the critical value of the control parameter $\alpha_{c}^{G L S}$ defined as $P\left(\sigma_{c}\left(\alpha_{c}^{G L S}\right)\right)=p_{c}$ a spanning cluster occurs, which has a fractal structure, the average size of finite clusters has a maximum at the critical point, and the cluster size distribution exhibits gap scaling [29]. However, this percolation is not related to the point of failure of the GLS bundle; the analogy to percolation is based purely on geometrical properties without any physical relevance.

Figure 18 shows that for localized load sharing the phase transition occurs when the damage fraction $p_{b}^{L L S}$ reaches the critical percolation probability $p_{c}$ of the corresponding lattice type. Due to the very localized load sharing, only short-range correlations arise in the system which are further moderated by the finite load-bearing capacity of broken fibers. Hence, in the vicinity of the transition point $p_{b}^{L L S}\left(\alpha_{c}\right) \approx p_{c}$ holds and the evolution of the microstructure of damage shows strong analogy to percolation lattices. It can be seen in Table I that the critical exponents of the plastic fiber bundle model are slightly different from the corresponding exponents of percolation; furthermore, the usual scaling relations of percolation critical exponents [29] are not fulfilled within the error bars. It has been shown for percolation that correlated occupation probabilities lead to the same critical behavior as random percolation when the correlations are short ranged $[38,39]$; however, long-range correlations result in changes of the critical exponents [38]. It is interesting to note that the value of the correlation length exponent $\nu$ of the PFBM is smaller than the value of random percolation which is consistent with the presence of relevant correlations [38]. We would like to emphasize that contrary to global load sharing, this percolationlike transition has important physical consequences on the behavior of the fiber bundle. The failure process of the bundle is dominated by the competition of fiber 


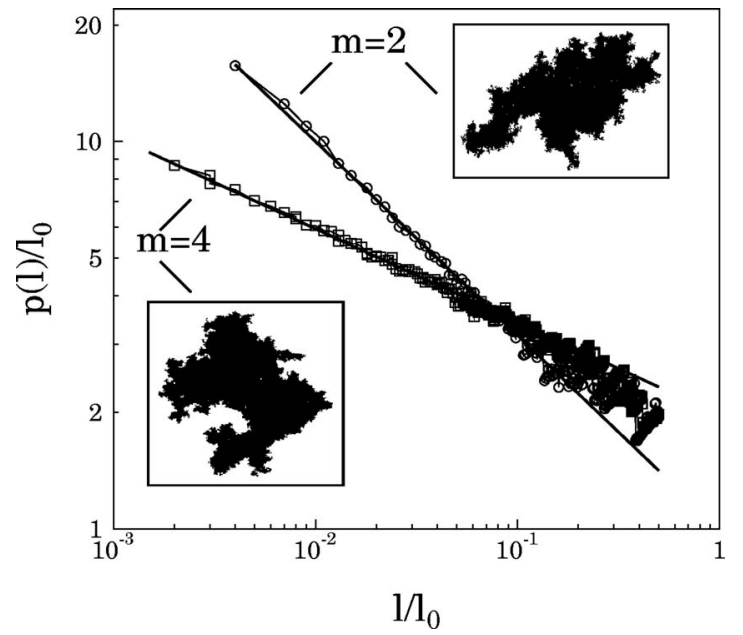

FIG. 19. Structure of the spanning cluster at two different disorder strengths in a lattice of size $L=401$. The perimeter length $p(l)$ of the cluster is plotted as function of the length $l$ of the yardstick normalized by the side length $l_{0}$ of the inscribing square. The insets present the clusters analyzed.

breaking by local stress enhancement due to load redistribution and by local weakness due to disorder. Our detailed analysis revealed that the relative importance of the two effects is controlled by the parameter $\alpha$. Below the critical point $\alpha<\alpha_{c}$ high stress concentration can develop around cracks so that the failure of the bundle occurs due to localization. Above the critical point $\alpha \geqslant \alpha_{c}$ the macroscopic response of the LLS bundle becomes practically identical with the GLS constitutive behavior, showing the dominance of disorder. It is important to note that the size distribution of bursts of simultaneously failing fibers becomes a power law at the critical point $\alpha_{c}$ with an exponent $\mu$ equal to the value recently predicted for GLS bundles of so-called critical failure threshold distributions $[9,10]$. This can be explained such that the large avalanches of the power-law distribution occurring in the plastic fiber bundle model at $\alpha_{c}$ (see Fig. 11) are dominated by the strong fibers of the bundle whose strength distribution is close to critical $[9,10]$.

The structure of the spanning cluster of the LLS bundle formed at the critical point $\alpha_{c}$ has also remarkable features different from the spanning cluster of percolation [29]. The insets of Fig. 19 present representative examples of the spanning cluster of a system of size $L=401$ at two different disorder strengths. It can be observed that the clusters are compact, they practically do not have holes, and there are no islands of unbroken fibers in the interior of the cluster. This structure is a direct consequence of the merging of growing compact clusters where especially large stress concentrations arise between the cluster surfaces, breaking the fibers and filling the holes in the spanning cluster. We note that in the limiting case of very strong disorder a small amount of intact fibers may survive dispersed over the spanning cluster. The result implies that the fractal dimension of the spanning cluster of the LLS bundle is 2, which should be compared to the corresponding value of random percolation $D=91 / 48 \approx 1.896$ where a finite amount of holes exists [29] even for short-range correlated occupation probabilities [39].
The perimeter of the spanning cluster, however, has a fine structure; i.e., it has a large number of peninsulas and valleys of all sizes. To reveal the structure of the perimeter, we measured its length $p(l)$ as a function of the length of the yardstick $l$. It can be seen in Fig. 19 that $p(l)$ shows a power-law dependence on $l$ over almost two decades:

$$
p(l) \sim l^{-\delta_{p}},
$$

where the value of the exponent proved to be $\delta_{p}=0.5 \pm 0.03$ for a Weibull distribution of fiber strength with $m=2$. The power law, Eq. (21), indicates that the perimeter line is a fractal with a dimension $D_{p}=1+\delta_{p}=1.5 \pm 0.03$. The upper bound of the scaling range in Fig. 19 can be attributed to the characteristic size of peninsulas of the spanning cluster, over which the rough structure of the perimeter disappears. Numerical calculations revealed that the fractal dimension of the cluster surface $D_{p}$ is not universal; i.e., it depends on the strength of disorder of the breaking thresholds. The insets of Fig. 19 illustrate that a lower amount of disorder gives rise to a more regular, smoother cluster surface characterized by a lower value of $D_{p}$. For the Weibull index $m=4$ we obtained $D_{p}=1.24 \pm 0.05$, which is significantly smaller than the corresponding value of $m=2$. The surface of damage clusters should be compared to the hull of the spanning cluster of percolation with the fractal dimension $D_{p}=7 / 4=1.75$ [40] (see also Table I).

\section{SUMMARY}

We introduced a fiber bundle model where failed fibers retain a fraction $0 \leqslant \alpha \leqslant 1$ of their failure load. The value of the parameter $\alpha$ interpolates between the perfectly rigid failure $\alpha=0$ and perfect plasticity $\alpha=1$ of fibers. We carried out a detailed study of the effect of the finite load-bearing capacity of fibers on the microscopic damage process and macroscopic response of fiber bundles considering both global and local load sharing for the load redistribution after fiber failure. Analytic calculations and computer simulations revealed that under global load sharing the macroscopic constitutive behavior of the interface shows a transition to perfect plasticity when $\alpha \rightarrow 1$, where the yield stress proved to be the average fiber strength. Approaching the state of perfect plasticity, the size distribution of bursts has a crossover from the mean-field power-law form of exponent 2.5 to a faster exponential decay.

When the load sharing is localized it is found that the load carried by the broken fibers has a stabilizing effect on the bundle; i.e., it lowers the stress concentration around clusters of failed fibers which has important consequences on the microscopic process of fracture and on the macroscopic response of the bundle. Extensive numerical calculations showed that at a specific value $\alpha_{c}$ a very interesting transition occurs from a phase where macroscopic failure emerges due to stress enhancement around failed regions leading to localization, to another phase where the disordered fiber strength plays the dominating role in the damage process.

On the macrolevel, below the critical point $\alpha<\alpha_{c}$ the fiber bundle shows a brittle response; i.e., the macroscopic failure is preceded by a weak nonlinearity, while for $\alpha \geqslant \alpha_{c}$ 
the constitutive behavior of the LLS bundle becomes practically identical with the GLS counterpart. Analyzing the evolution of the microstructure of damage with increasing $\alpha$, the transition proved to be continuous, analogous to percolation. Computer simulations revealed that the avalanche-size distribution of fiber breakings becomes a power law at the critical point with a universal exponent equal to the mean-field exponent of bundles with critical strength distributions. The spanning cluster of failed fibers formed at the transition point proved to be compact with a fractal boundary whose dimension increases with the amount of disorder. The critical value $\alpha_{c}$ is not universal; besides the lattice structure, it also depends on the strength of disorder.

The plastic fiber bundle model can be relevant for the shear failure of interfaces where failed surface elements can remain in contact, still transmitting load. Such glued interfaces of solids typically occur in fiber composites, where fibers are embedded in a matrix material. The finite loadbearing capacity of failed elements of the model can account for the frictional contact of debonded fiber-matrix interfaces and also for plastic behavior of the components.

\section{ACKNOWLEDGMENTS}

This work was supported by the Collaborative Research Center SFB 381. F.K. acknowledges financial support of Research Contract Nos. NKFP 3A-043/2004, OTKA M041537, and T049209 and of the György Békésy Foundation of the Hungarian Academy of Sciences.
[1] Statistical Models for the Fracture of Disordered Media, edited by H. J. Herrmann and S. Roux (Elsevier, Amsterdam, 1990).

[2] B. K. Chakrabarti and L. G. Benguigui, Statistical Physics of Fracture and Breakdown in Disordered Systems (Oxford University Press, New York, 1997).

[3] H. E. Daniels, Proc. R. Soc. London, Ser. A 183, 405 (1945).

[4] D. G. Harlow and S. L. Phoenix, J. Compos. Mater. 12, 314 (1978).

[5] W. A. Curtin, Phys. Rev. Lett. 80, 1445 (1998).

[6] F. Kun and H. J. Herrmann, J. Mater. Sci. 35, 4685 (2000).

[7] S. Pradhan and B. K. Chakrabarti, Int. J. Mod. Phys. B 17, 5565 (2003).

[8] M. Kloster, A. Hansen, and P. C. Hemmer, Phys. Rev. E 56, 2615 (1997).

[9] S. Pradhan, A. Hansen, and P. C. Hemmer, Phys. Rev. Lett. 95, 125501 (2005).

[10] S. Pradhan and A. Hansen, Phys. Rev. E 72, 026111 (2005).

[11] R. C. Hidalgo, F. Kun, and H. J. Herrmann, Phys. Rev. E 64, 066122 (2001).

[12] J. V. Andersen, D. Sornette, and K. T. Leung, Phys. Rev. Lett. 78, 2140 (1997).

[13] Y. Moreno, J. B. Gomez, and A. F. Pacheco, Phys. Rev. Lett. 85, 2865 (2000).

[14] F. Kun, Y. Moreno, R. C. Hidalgo, and H. J. Herrmann, Europhys. Lett. 63, 347 (2003).

[15] P. Bhattacharyya, S. Pradhan, and B. K. Chakrabarti, Phys. Rev. E 67, 046122 (2003).

[16] S. Pradhan and B. K. Chakrabarti, Phys. Rev. E 65, 016113 (2002).

[17] G. G. Batrouni, A. Hansen, and J. Schmittbuhl, Phys. Rev. E 65, 036126 (2002).

[18] S. Zapperi, H. J. Herrmann, and S. Roux, Eur. Phys. J. B 17, 131 (2000).

[19] J. Knudsen and A. R. Massih, Phys. Rev. E 72, 036129 (2005).

[20] A. Delaplace, S. Roux, and G. Pijaudier-Cabot, J. Eng. Mech.
127, 646 (2001).

[21] F. Raischel, F. Kun, and H. J. Herrmann, Phys. Rev. E 72, 046126 (2005).

[22] D. Sornette, J. Phys. A 22, L243 (1989).

[23] F. Kun, S. Zapperi, and H. J. Herrmann, Eur. Phys. J. B 17, 269 (2000).

[24] P. C. Hemmer and A. Hansen, J. Appl. Mech. 59, 909 (1992).

[25] A. Hansen and P. C. Hemmer, Phys. Lett. A 184, 394 (1994).

[26] R. C. Hidalgo, Y. Moreno, F. Kun, and H. J. Herrmann, Phys. Rev. E 65, 046148 (2002).

[27] S. Zapperi, P. Ray, H. E. Stanley, and A. Vespignani, Physica A 270, 57 (1999a).

[28] S. Zapperi, P. Ray, H. E. Stanley, and A. Vespignani, Phys. Rev. E 59, 5049 (1999b).

[29] D. Stauffer and A. Aharony, Introduction to Percolation Theory (Taylor \& Francis, London, 1992).

[30] M. E. J. Newman and G. T. Barkema, Monte Carlo Methods in Statistical Physics (Clarendon Press, Oxford, 1999).

[31] P. V. V. Nukala, S. Simunovic, and S. Zapperi, J. Stat. Mech.: Theory Exp. ( 2004)P08001.

[32] S. Zapperi, P. Ray, H. E. Stanley, and A. Vespignani, Phys. Rev. Lett. 78, 1408 (1997).

[33] A. Hansen, S. Roux, and H. J. Herrmann, J. Phys. (Paris) 50, 733 (1989).

[34] G. G. Batrouni and A. Hansen, Phys. Rev. Lett. 80, 325 (1998).

[35] P. Ray and G. Date, Physica A 229, 26 (1996).

[36] S. Roux, A. Hansen, H. Herrmann, and W. Guyon, J. Stat. Phys. 52, 237 (1988)

[37] G. A. D' Addetta, F. Kun, E. Ramm, and H. J. Herrmann, in Continuous and Discontinuous Modelling of CohesiveFrictional Materials, edited by P. Vermeer et al. (SpringerVerlag, Berlin, 2001).

[38] A. Weinrib, Phys. Rev. B 29, 387 (1984).

[39] S. R. Anderson and F. Family, Phys. Rev. A 38, 4198 (1988).

[40] H. Saleur and B. Duplantier, Phys. Rev. Lett. 58, 2325 (1987). 